\title{
The choice of seed tracking method influenced fate of beech seeds dispersed by rodents
}

\author{
Aleksandra Wróbel · Rafał Zwolak
}

Received: 28 December 2012/ Accepted: 4 February 2013/Published online: 15 February 2013

(C) The Author(s) 2013. This article is published with open access at Springerlink.com

\begin{abstract}
We tracked seeds of European beech (Fagus sylvatica) dispersed by rodents in Gorzowska Forest (western Poland). We used two seed labeling methods, marking with UV-fluorescent powder and with plastic tags, to test whether using different marking methods influences results of seed tracking. The removal rates did not differ among seeds marked with UV-powder, seeds labeled with tags, and unmanipulated seeds. We found $78 \%$ of removed seeds marked with tags, but only $25 \%$ of UV-marked seeds. The consumption rates of tagged and UV-marked seeds were dramatically different: rodents ate $83 \%$ of the former and $26 \%$ of the later. The average dispersal distance was larger for seeds marked with UV-powder than for tagged seeds. Our findings suggest that the choice of seed tracking method might influence results of seed dispersal studies.
\end{abstract}

Keywords Animal dispersed seeds .

Seed-caching rodents $\cdot$ Seed fate

\section{Introduction}

Many plant species rely on scatterhoarding animals for seed dispersal. The net effect of scatterhoarders on

A. Wróbel · R. Zwolak $(\bowtie)$

Department of Systematic Zoology, Institute of Environmental Biology, Adam Mickiewicz University, Umultowska 89, 61-614 Poznan, Poland

e-mail: rafal.zwolak@gmail.com plant recruitment is influenced by multiple factors such as the number of seeds handled, probability of caching, place of seed deposition, or dispersal distance. Thus, thorough understanding of plant-scatterhoarder interactions requires establishing the fate of removed seeds. Accordingly, tracking of removed seeds is increasingly common in ecological studies.

Most popular seed tracking techniques include tag-marking, thread-marking, labeling with UVfluorescent powder, radioisotope tracking, and radiotelemetry methods (Forget and Wenny 2005). These methods differ in effectiveness (percentage of retrieved seeds) and costs, but it is usually assumed that the choice of seed marking technique does not influence seed fate. However, marking changes the appearance of seeds (perhaps with the exception of radioisotope labeling). Given numerous research indicating that seed characteristics such as size, weight, chemical composition, or odor influence animal decisions (Duncan et al. 2002; Xiao et al. 2005; Wang and Chen 2009), it is possible that seed marking affects scatterhoarder behavior, but testing for such effects is uncommon (see Xiao et al. 2006; Yi et al. 2008; Hirsch et al. 2012).

We tracked rodent-dispersed European beech (Fagus sylvatica) seeds by two simple and widely used methods of labeling seeds: with plastic tags and UV-powder (Forget and Wenny 2005: beech seeds weigh about $0.3 \mathrm{~g}$ and are too small to use radiotelemetry-based methods). Using two methods simultaneously enabled us to test whether the fate of rodent-dispersed seed differed depending on the marking technique. 


\section{Methods}

The study was conducted in September and October 2010 in Gorzowska Forest (western Poland, 52.77N, 15.07E), at six beech stands located $0.2-2 \mathrm{~km}$ from one another. Rodent communities at the study site were dominated by the yellow-necked mouse Apodemus flavicollis and the bank vole Myodes glareolus (Zwolak, unpublished).

Because beech produced few seeds in 2010, the experiments were conducted with beech seeds obtained from the seed storage in Forestry Office Gryfino. Seeds were handled using rubber gloves to avoid scent contamination (Duncan et al. 2002).

\section{Seed preparation}

We assigned 384 seeds for each of the three treatments: plastic tag tracking, fluorescent powder tracking, and unmanipulated control. Labeling seeds with tags consisted of drilling $0.8-\mathrm{mm}$ holes through cotyledons and tying steel wire (100-mm length, 0.2-mm diameter) with a red plastic tag $(20 \times 40 \mathrm{~mm})$. On average, the wire and tag weighted $0.141 \mathrm{~g}(\mathrm{SD}=0.004 \mathrm{~g})$. The tagged seeds were put out in Petri dishes (four per dish).

For UV-powder tracking, four seeds in a Petri dish were placed in the middle of an aluminum tray $(24 \times 31 \mathrm{~cm})$ lined with sandpaper (Tomback et al. 2005). The seeds, the Petri dish, and the sandpaper were coated with fluorescent powder (Stanimex $^{\mathrm{TM}}$, Lublin, Poland). After the first night of tracking, the trays were cut to flatten them out because raised rims discouraged rodents from accessing seeds. The data on removal of UV-marked seeds during that night were excluded from the analyses.

The control seeds were also put out in Petri dishes (four per dish).

Field study

At each site, the Petri dishes with seeds (hereafter "seed depots") were arranged in three 60-m transects located $30 \mathrm{~m}$ from one another. Each transect consisted of four seed depots, thus there were 12 depots per site. At each site, we randomly assigned four depots to each treatment: tagging, UV-marking, and control.

We conducted four nights of tracking at each study site, each night using a new batch of seeds. The seeds were put out at dusk (19:00-21:00), tracking began at 3:00-4:00 and lasted until dawn $(\sim 7: 00)$. We used flashlights to find tagged seeds, and UV-lights to detect seeds marked with fluorescent powder. If marked seeds were found, we measured their distance from the depot of origin (as denoted on seed tags or, in the case of UV-marked seeds, determined by following fluorescent trails), categorized their fate as consumed, left on surface, or buried (in soil or leaves), and removed them from site. Seeds buried in topsoil were found because their tags were left on surface (in the case of tagged seeds) or because digging rodents left blotches of fluorescent powder (in the case of UV-marked seeds). However, some seeds were probably larderhoarded in deep burrows. We were unable to find such seeds, regardless of the marking method used.

Statistical analyses

We analyzed whether the seed tracking methods differed with regard to (i) probability of seed removal, (ii) probability of finding removed seeds, (iii) probability that removed seeds were found consumed, (iv) probability that removed but uneaten seeds were found buried, and (v) removal distance. The analyses were conducted in $\mathrm{R}$ using generalized linear mixed models. In analyses (i)-(iv), we used binomial error distribution and in analysis (v) Gaussian error distribution with removal distances log-transformed. Testing for statistical significance was conducted with likelihood ratio tests (analyses i-iv) and $t$-statistics (analysis v, $P$-values obtained with Markov chain Monte Carlo sampling).

In each model, we entered seed labeling method as fixed effect, whereas site, seed depot, and night of tracking were entered as random effects. The only exception was seed removal analysis. Since fate of all seeds within a depot was usually the same (see Results section), the appropriate response variable was depot rather than single seed with fixed effect of labeling method and random effects of site and night.

\section{Results}

Seed removal

Rodents removed seeds from $59 \%$ of control trays, $52 \%$ of trays containing tagged seeds, and $50 \%$ of 
trays with UV-marked seeds. The differences among treatment were not significant $\left(\chi^{2}=2.37\right.$, df $=2$, $P=0.30$ ). Once a tray was discovered, rodents usually removed all seeds: this occurred with all exploited trays containing control seeds (56 out of 56), 46 out of 50 exploited tagged seed trays, and 40 out of 42 exploited trays with UV-marked seeds.

\section{Retrieval of removed seeds}

We found $78 \%$ of removed seeds marked with tags and $25 \%$ of UV-marked seeds $\left(\chi^{2}=50.25, \mathrm{df}=1\right.$, $P<0.001)$.

\section{Consumption and caching}

Estimated consumption probability was considerably higher for tagged seeds than for UV-marked seeds (83 vs. $26 \% ; \chi^{2}=9.31, \mathrm{df}=1, P=0.002$ ). For seeds that were found uneaten $(n=63)$, the estimated burial probability did not differ between the seed tracking methods (tagging: $71 \%$; UV-powder: $87 \% ; \chi^{2}=0.55$, df $=1, P=0.46$ ).

\section{Removal distance}

Seeds marked with UV-powder were moved farther than tagged seeds (Fig. 1a) although the furthest dispersal distances were recorded for seeds with tags (outliers in Fig. 1a). However, the differences were a consequence of different fates of UV-marked and tagged seeds. Seeds that were removed and left uneaten (buried or left on surface) were moved farther than seeds that were removed and consumed (buried vs. eaten: $t=-8.41, P<0.001$; left on surface vs. eaten: $t=4.68, P<0.001$; Fig. 1b). In addition, buried seeds tended to be moved farther than unburied seeds ( $t=1.78, P=0.077$; Fig. 1b). After these effects were accounted for, marking method was not a significant predictor of dispersal distance (likelihood ratio test, $\chi^{2}=0.00$, df $=1, P>0.99$ ).

\section{Discussion}

The two seed tracking methods differed in efficiency (percentage of recovered seeds), but, most importantly,

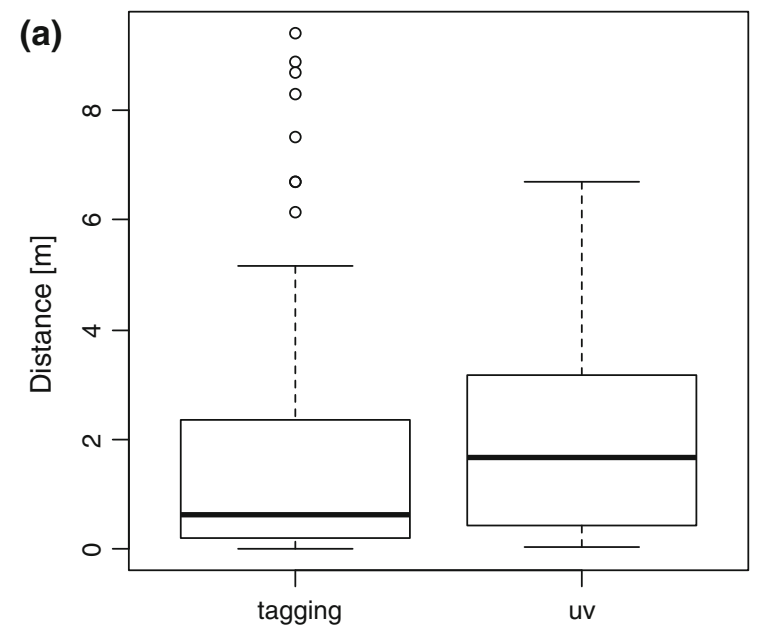

Seed tracking method

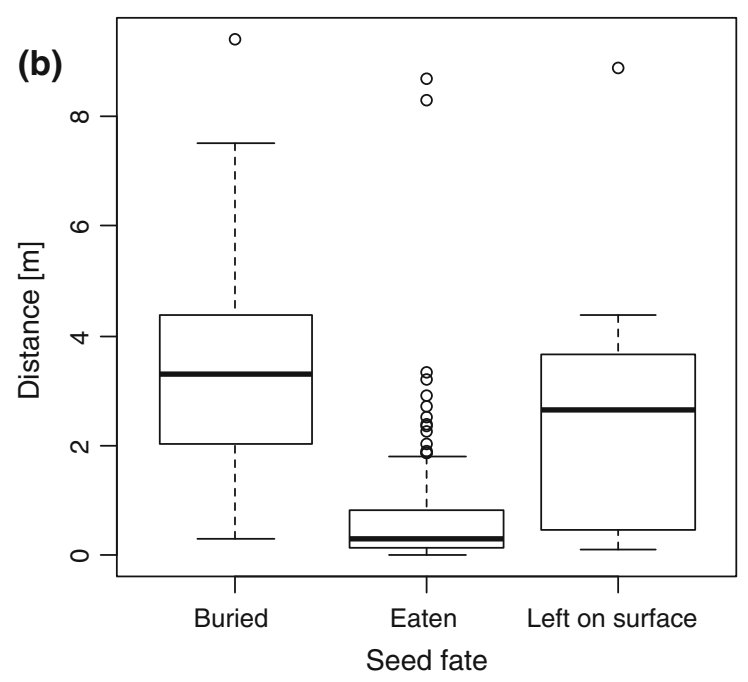

Fig. 1 Translocation distances, categorized according to seed tracking method (a) and fate of removed seeds (b). Boxes denote 25th, 50th, and 75th percentiles, whiskers denote the farthest data points within 1.5 interquartile range and the open circles denote data points beyond the 1.5 interquartile range

appeared to influence the fate of seeds removed by rodents. Tagged seeds were usually consumed, whereas UV-marked seeds were usually left intact, either on the litter surface or buried. These findings suggest that inferences from seed tracking studies might depend on the seed labeling method used, thus comparisons across studies that used different methods must be made cautiously. Moreover, our results emphasize the need for testing seed-tracking methods for side effects on disperser behavior. Below, we discuss differences between the tested methods in more detail. 
High recovery rate is one of the most desired properties of seed tagging methods. The retrieval rate equaled $97 \%$ for radio-tracked seeds (Hirsch et al. 2012), 51-91\% for radioisotope-labeled seeds (Vander Wall 1994), 54-87\% for seeds with tin-tags on a wire (Xiao et al. 2005), and $26 \%$ for spool-and-line technique (Yasuda et al. 1991). Compared with previous research, the percentage of retrieved seeds in our study was relatively high for tagged seeds, but low for UV-marked seeds.

Besides having high recovery rate, good seedtracking methods should not influence disperser behavior (Forget and Wenny 2005). However, testing for such effects is challenging and therefore is usually limited to the seed removal stage. Typically, seed removal is unaffected by marking (e.g., Li and Zhang 2003; Hirsch et al. 2012; but see Xiao et al. 2006), suggesting that removal rates are robust to changes in seed appearance. On the other hand, clue as subtle as human scent left when handling seeds influenced their removal (Duncan et al. 2002), thus this robustness is somewhat surprising.

In our study, seed marking did not affect removal rates, even though we used different substrates: UV-marked seeds were placed on trays lined with sandpaper, whereas the tagged and control seeds were not. However, once the seeds were removed, they had higher chance of consumption when they were marked with tags than with UV-powder. Therefore, similar removal rates of marked and unmanipulated seeds cannot be taken as evidence that the fate and movements of seeds are unaffected by marking.

Higher consumption rates of tagged than UV-marked seeds can have several explanations. First, holes drilled in seeds to attach tags might be used by rodents as a cue for insect infestation. Infested seeds are more likely to be eaten and less likely to be cached than sound seeds (Steele et al. 1996). However, infested nuts often have reduced removal rates (Perea et al. 2012), whereas removal rates of tagged seeds appeared unaffected.

Second, UV-powder (tasteless, but not completely odorless) might deter rodents from eating seeds. However, if this was the case, removal rates of UV-marked seeds should also be affected.

Third, seeds with tags might be more difficult to transport than control or UV-marked seeds. Compared with consumption, caching usually takes place farther from seed source (Li and Zhang 2003; see also
Results section of this study), thus if it is difficult to transport seeds over long distances, they might be eaten rather than cached.

Finally, it might be more difficult to find consumed UV-marked seeds than consumed tagged seeds. Nevertheless, this explanation seems unlikely. Intact and consumed UV-marked seeds were similarly visible because when rodents consume beech nuts, seed shells (coated with UV-powder) are left largely intact. However, even if the difference in consumption rates of seeds coated with UV-powder and tagged seeds was caused by differences in their detectability, it does not change the conclusion that by different seed marking methods might produce different results.

We were unable to test which of the two seed labeling methods had stronger influence on rodent behavior. In general, it is virtually impossible to compare post-removal fates of marked and unmanipulated seeds because unlabeled seeds cannot be tracked. Probably the best solution would be to use a method that is least likely to influence animal behavior as a reference (e.g., radioactive labeling, although this method might not be considered optimal due to possible adverse effects to environment and animals).

Our study indicates that the choice of seed labeling method might influence results of seed tracking. Therefore, we suggest choosing seed marking methods that are as subtle as possible. Even then, most seed tracking results should be regarded as an index of seed fate rather than a perfect reflection of seed dispersal by animals. In other words, the fate of marked seeds probably correlates with the fate of unmarked seeds rather than provides its absolute measure. However, this is not necessarily a problem since in many cases, we are interested in relative comparisons (e.g., between mast and non-mast years: Xiao et al. 2005; between seeds of different size and chemical composition: Wang and Chen 2009) rather than absolute measures of seed dispersal.

Acknowledgments We thank Forest District Bogdaniec for permission to conduct this study. Michał Bogdziewicz, Sylwia Dziemian, and Anna Osiecka helped with field work. This study was conducted while R. Zwolak was supported by a MNiSW Grant NN304391537 and a FNP fellowship HOM/2009/16B.

Open Access This article is distributed under the terms of the Creative Commons Attribution License which permits any use, distribution, and reproduction in any medium, provided the original author(s) and the source are credited. 


\section{References}

Duncan RS, Wenny DG, Spritzer MD, Whelan CJ (2002) Does human scent bias seed removal studies? Ecology 83: 2630-2636

Forget PM, Wenny D (2005) How to elucidate seed fate? A review of methods used to study seed removal and secondary seed dispersal. In: Forget PM, Lambert J, Hulme PE, Vander Wall SB (eds) Seed fates, predation, dispersal and seedling establishment. CABI Publishing, Wallingford, pp 379-393

Hirsch BT, Kays R, Jansen PA (2012) A telemetric thread tag for tracking seed dispersal by scatter-hoarding rodents. Plant Ecol 213:933-943

Li HJ, Zhang ZB (2003) Effect of rodents on acorn dispersal and survival of the Liaodong oak (Quercus liaotungensis Koidz.). For Ecol Manag 176:387-396

Perea R, San Miguel A, Martínez-Jauregui M, Valbuena-Carabaña M, Gil L (2012) Effects of seed quality and seed location on the removal of acorns and beechnuts. Eur J For Res 131:623-631

Steele MA, Hadj-Chikh LZ, Hazeltine J (1996) Caching and feeding decisions by Sciurus carolinensis: responses to weevil-infested acorns. J Mammal 77:305-314
Tomback DF, Schoettle AW, Chevalier KE, Jones CA (2005) Life on the edge for limber pine: seed dispersal within a peripheral population. Ecoscience 12:519-529

Vander Wall SB (1994) Seed fate pathways of antelope bitterbrush: dispersal by seed-caching yellow pine chipmunks. Ecology 75:1911-1926

Wang B, Chen J (2009) Seed size, more than nutrient or tannin content, affects seed caching behavior of a common genus of Old World rodents. Ecology 90:3023-3032

Xiao Z, Zhang Z, Wang Y (2005) The effects of seed abundance on seed predation and dispersal by rodents in Castanopsis fargesii (Fagaceae). Plant Ecol 177:249-257

Xiao Z, Jansen PA, Zhang Z (2006) Using seed-tagging methods for assessing post-dispersal seed fate in rodent-dispersed trees. For Ecol Manag 223:18-23

Yasuda M, Nagagoshi N, Takahashi FR (1991) Examination of the spool-and-line method as a quantitative technique to investigate seed dispersal by rodents. Jpn J Ecol 41: 257-262

Yi X, Xiao Z, Zhang Z (2008) Seed dispersal of Korean pine Pinus koraiensis labeled by two different tags in a northern temperate forest, northeast China. Ecol Res 23:379-384 